\title{
Decomposition of I nterference Hyperspectral Images Based on Split Bregman Iteration
}

\author{
Jia Wen $^{1 *}$, Lei Geng ${ }^{1}$, Cailing Wang ${ }^{2}$ \\ ${ }^{1}$ Tianjin Key Laboratory of Optoelectronic Detection Technology and Systems, School of Electronics and \\ Information Engineering, Tianjin Polytechnic University, Tianjin, 300387, China \\ [e-mail: Dr_JWen@qq.com] \\ ${ }^{2}$ College of Computer Science, Xi'an Shiyou University, Xi'an, 710065, China \\ [e-mail: azering@163.com] \\ *Corresponding author: Jia Wen
}

Received January 1, 2018; revised February 23, 2018; accepted March 2, 2018; published July 31, 2018

\begin{abstract}
Images acquired by Large Aperture Static Imaging Spectrometer (LASIS) exhibit obvious interference stripes, which are vertical and stationary due to the special imaging principle of interference hyperspectral image (IHI) data. As the special characteristics above will seriously affect the intrinsic structure and sparsity of IHI, decomposition of IHI has drawn considerable attentions of many scientists and lots of efforts have been made. Although some decomposition methods for interference hyperspectral data have been proposed to solve the above problem of interference stripes, too many times of iteration are necessary to get an optimal solution, which will severely affect the efficiency of application. A novel algorithm for decomposition of interference hyperspectral images based on split Bregman iteration is proposed in this paper, compared with other decomposition methods, numerical experiments have proved that the proposed method will be much more efficient and can reduce the times of iteration significantly.
\end{abstract}

Keywords: IHI (interference hyperspectral images), split Bregman iteration, sparse representation, total variation

This work was supported by the Tianjin Natural Science Foundation (No. 17JCQNJC01400), National Natural Science Foundation of China (Grant No. 61401439, 61601323) and Tianjin Science and Technology Major Projects and Engineering (17ZXSCSY00090). 


\section{Introduction}

Interference hyperspectral imaging is a powerful technology in the field of remote sensing, which can get the spectral and spatial information of the observed targets. The above technology has been widely used in many fields, such as geology, military, meteorology and so on. In the "Chang'E" lunar exploration satellite and environmental monitoring satellite, the interference hyperspectral spectrometer has both been successfully equipped, and has also become the research focus in the recent years. As the reason that the special imaging principle of interference hyperspectral technology is light interference, lots of interference stripes exist in each frame of interference hyperspectral image (IHI), which will destroy the image's intrinsic structure and sparsity severely. As the above reason, it is difficult to find an existing orthonormal basis to sparsely represent the IHI data. In addition, due to the huge amount and high resolution of the IHI, over-complete dictionary learning for IHI will waste too much time and make the whole process inefficient.

In order to make the IHI data be easily sparsely represented, the idea of separating the interference stripe layers (layers containing only interference stripes) from the background layers (layers containing only natural information without any interference stripe) is taken into account, and many state-of-the-art algorithms of image layers decomposition for IHI data have been proposed in recent years.

Morphological component analysis (MCA) theory is adopted for the image layer decomposition of IHI data, which is proposed in 2015 [1]; IMCA(Improved MCA) algorithm is proposed in [2], which is improved according to the IHI data's special characteristics; In [3], idea of MCA and Total Variation (TV) is combined and an IMT (Improved MCA-TV) algorithm is proposed for decomposition of the IHI data. The decomposition models proposed in [1-3] could realize the image layer decomposition for IHI data successfully, but all of the proposed models have a common defect: low efficiency. All of the above algorithms need too many times of iteration and spend too much time, which will affect the efficiency of decomposition seriously. However, a decomposed model of interference hyperspectral images based on split Bregman iteration is proposed in this paper, which can realize the IHI image decomposition with high efficiency due to its simple linear operations.

The paper will be organized as follows: Characteristic of the IHI data will be introduced in section 2. Section 3 will introduce the theory of split Bregman iteration. The decomposition model based on split Bregman iteration for IHI data will be proposed in section 4. Experiments and analysis will be given in section 5 and the conclusion and prospect will be given in section 6.

\section{Characteristic of Interference Hyperspectral Images}

The imaging principle and equivalent optical path of the interference imaging spectrometer have been described in [1-3]. Fig. 1 shows the equivalent optical path in lateral shearing interferometer. $S_{1}$ and $S_{2}$ are the two separated rays by the light from a ground point. In the interferometer, The OPD (Optical Path Difference) of point $O$ in CCD detector is zero. FTL (Fourier transform lens) is the main imaging equipment, $f_{F T L}$ is the focus of Fourier lens. 


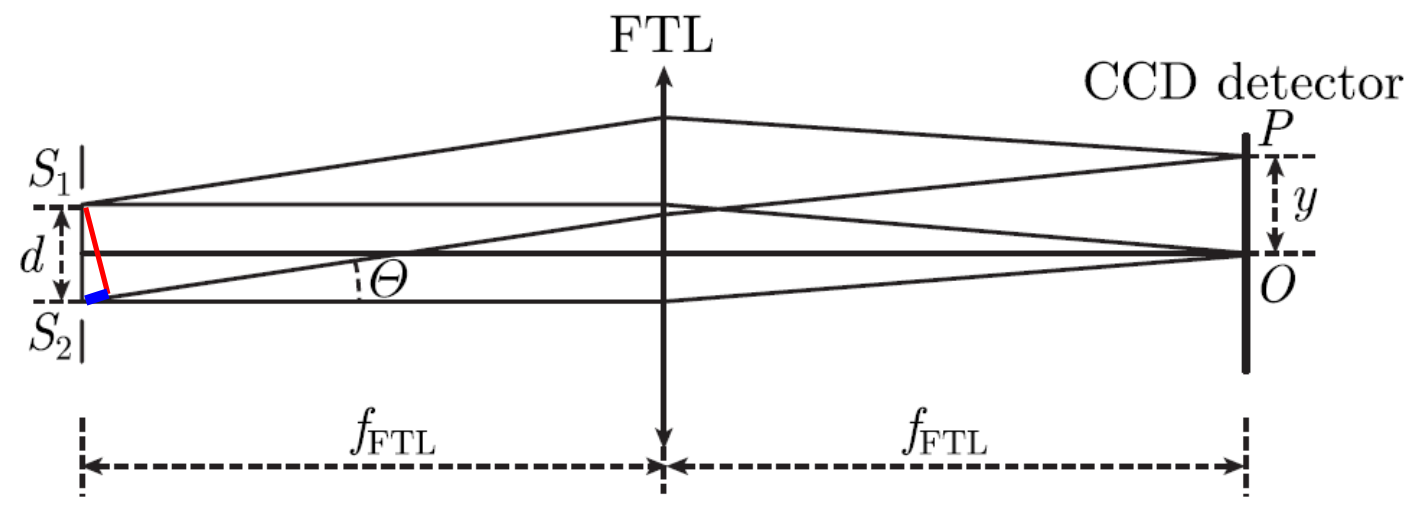

Fig. 1. The sketch map of the interference hyperspectral spectrometer

The OPD of point $P$ on the CCD is:

$$
x=d \sin \theta=y \frac{d}{f_{F T L}}
$$

The explanation of Formula (1) :As Fig. 1 shows, the distance between $P$ and $O$ on the CCD detector is $y$, so the value of $\sin \theta$ can approximately be calculated as $y / f_{F T L}$. A red line (which is vertical to the line $S_{2} P$ ) is added in Fig. 1, the angle between line $S_{1} S_{2}$ and the red line added are also $\theta$, so the OPD of point P (length of the blue line added in Fig. 1) can be calculated as Formula (1) shows.

According to the theory of Fourier transform, the interference curve can be expressed as:

$$
I(x)=\int_{k_{\min }}^{k_{\max }} B(k) e^{j 2 \pi k x} d k=\int_{k_{\min }}^{k_{\max }} B(k) e^{j 2 \pi k \frac{y d}{f_{F T L}}} d k
$$

In which $B(k)$ is the spectral distribution of source, $k_{\max }$ and $k_{\min }$ are the extremums of wavenumber, $x$ represents OPD of this interference curve. As the Fourier transform of $B(k)$ is a real and even function. So formula (2) equals to

$$
I(x)=\int_{k_{\min }}^{k_{\max }} B(k) \cos (2 \pi k x) d k=\int_{k_{\min }}^{k_{\max }} B(k) \cos \left(2 \pi k y \frac{d}{f_{F T L}}\right) d k
$$

Which is the mathematical model of the vertical interference stripes in the IHI data, and $I(x)$ will have different values at different OPD (which $x$ represents), that is the reason why the maximum amplitude of vertical interference stripes always exist at the same places.

The spectrum curve can be obtained by inverse Fourier transform of the interference curve according to the basic Fourier transform relationship. The inverse Fourier transform of 
formula (2) is:

$$
B(k)=\int_{0}^{\delta_{\mathrm{m}}} I(x) e^{-j 2 \pi k x} d x=\int_{0}^{\delta_{\mathrm{m}}} I(x) e^{-j 2 \pi k \frac{y d}{f_{F T L}}} d x
$$

$\delta_{\mathrm{m}}$ is the maximum OPD. The corresponding cosine transform is:

$$
B(k)=\int_{0}^{\delta_{\mathrm{m}}} I(x) \cos (2 \pi k x) d x=\int_{0}^{\delta_{\mathrm{m}}} I(x) \cos \left(2 \pi k y \frac{d}{f_{F T L}}\right) d x
$$

The main characteristics of IHI data are as follows:

First, there are vertical interference stripes in each frame of IHI. Second, the background of IHI has horizontal shift between frames.

Vertical interference stripes obviously exist in each frame of IHI data. LASIS (Large Aperture Static Imaging Spectrometer) IHI data sequences are shown in Fig. 2, in which vertical interference stripes can been obviously found in the left part of the LASIS IHI data. To obtain the spectrum information from the IHI data, post-processing of corresponding column extraction from LASIS IHI data should be done [4] as Fig. 3 shows, and LSMIS (Large Spatially Modulated Interference Spectral Image) IHI data will be gotten after the post-processing as Fig. 4 shows.

As Fig. 4 shows vertical interference stripes still exist in the LSMIS IHI data, while the background image of LSMIS is composed of the corresponding columns of each LASIS IHI data frame, so the horizontal pixels of background image almost have the same value in LSMIS IHI data. As the above reason, we can utilize the unidirectional characteristics to decompose the LSMIS data into two different image layers [3]: 1) background layer; 2) interference stripe layer. From the point of view in mathematics, the background layer will have lower values of total variation (which is the summation of the gradient absolutions) in horizontal direction, while the interference stripe layer will have lower values of total variation in vertical direction. The optimal result of the decomposition can be calculated using the regularized thought as follows:

$$
\begin{aligned}
& X_{B_{-} o p t}=\underset{X_{B}}{\arg \min } \frac{\lambda_{1}}{2}\left\|\nabla_{x} \otimes X_{B}\right\|_{2}^{2}+\frac{\lambda_{2}}{2}\left\|\nabla_{y} \otimes X_{I}\right\|_{2}^{2} \\
& =\underset{X_{B}}{\arg \min } \frac{\lambda_{1}}{2}\left\|\nabla_{x} \otimes X_{B}\right\|_{2}^{2}+\frac{\lambda_{2}}{2}\left\|\nabla_{y} \otimes\left(X-X_{B}\right)\right\|_{2}^{2} \\
& <=>\underset{X_{B}}{\arg \min } \frac{\lambda_{1}}{2}\left\|\nabla_{x} \otimes X_{B}\right\|_{2}^{2}+\frac{\lambda_{2}}{2}\left\|\nabla_{y} \otimes\left(X_{B}-X\right)\right\|_{2}^{2}
\end{aligned}
$$


where $X$ is the original LSMIS IHI data, $X_{B}$ is the background layer, $X_{I}=X-X_{B}$ is the interference stripe layer, $\lambda_{1}, \lambda_{2}$ are the regularized weights of the two layers respectively, $\otimes$ is the convolution operator, $\nabla_{x}, \nabla_{y}$ are horizontal and vertical gradient operators respectively, and the convolution with $\nabla_{x}$ and $\nabla_{y}$ denotes the total variation in horizontal and vertical direction respectively [5]. $X_{B_{-} \text {opt }}$ is the optimal result of $X_{B}$, which is the background layer of LSMIS IHI data, so $X_{B_{-} o p t}$ should not have too much difference with the original image $X$. As the above reason, fidelity term is added as the following formula (6) shows:

$$
X_{B_{-} o p t}=\underset{X_{B}}{\arg \min } \frac{1}{2}\left\|X-X_{B}\right\|_{2}^{2}+\frac{\lambda_{1}}{2}\left\|\nabla_{x} \otimes X_{B}\right\|_{2}^{2}+\frac{\lambda_{2}}{2}\left\|\nabla_{y} \otimes\left(X_{B}-X\right)\right\|_{2}^{2}
$$

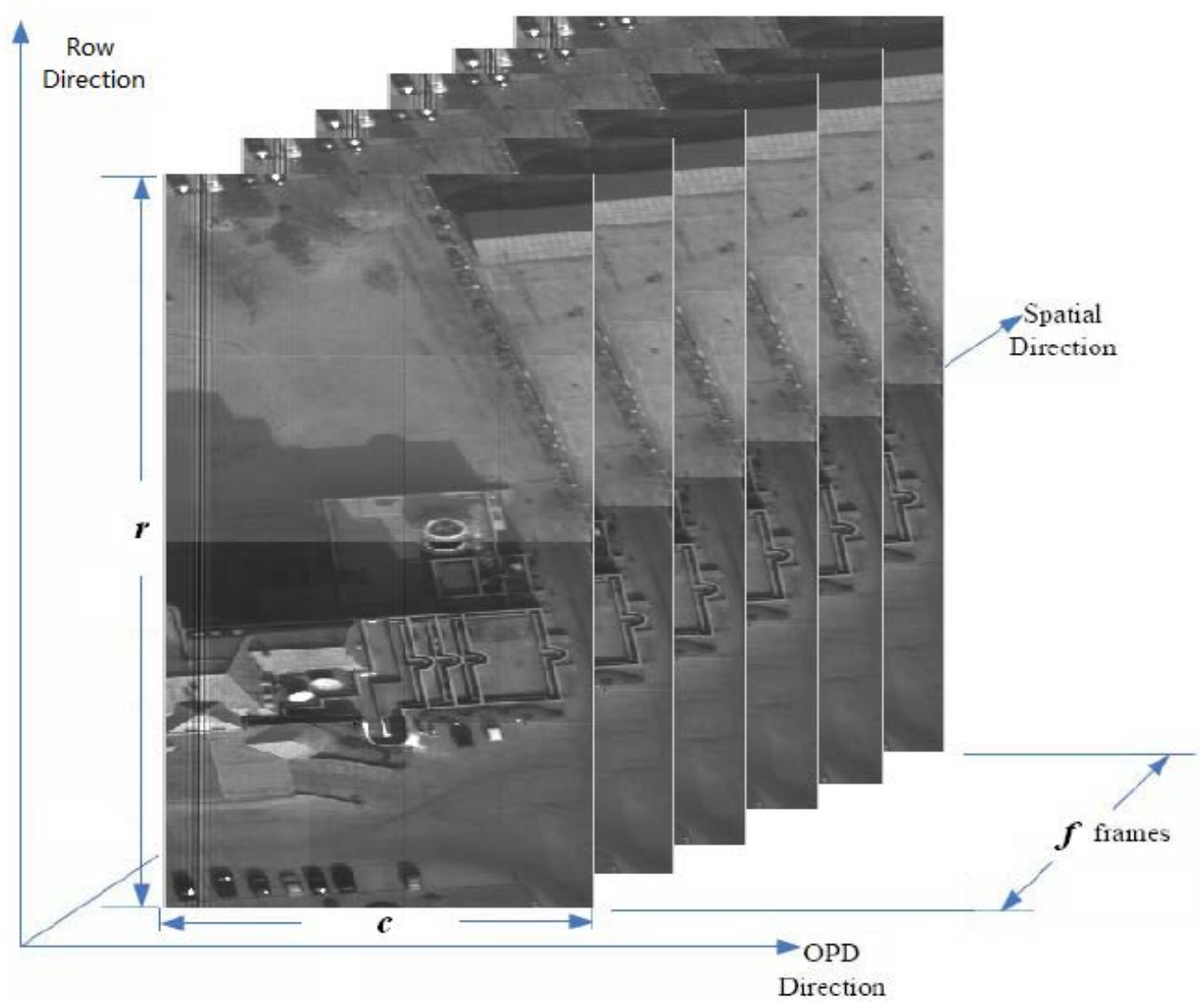

Fig. 2. LASIS interference hyperspectral image sequences

\section{Introduction of Split Bregman Iteration}

Split Bregman iteration [6] is an efficient algorithm to get the optimal solution of the following inverse problem: 


$$
\min _{u} E(u)+\lambda H(u)
$$

where $E(u)$ and $H(u)$ are the convex functions of fidelity term and regular term respectively. Formula (8) is adopted to solve the formula (7) as the following shows:

$$
u^{k+1}=\min _{u} D_{E}^{P}\left(u, u^{k}\right)+\lambda H(u)
$$

where $D_{E}^{P}\left(u, u^{k}\right)$ is defined as the Bregman distance between $u$ and $u^{k}$ :

$$
D_{E}^{P}\left(u, u^{k}\right)=E(u)-E\left(u^{k}\right)-\left\langle p, u-u^{k}\right\rangle
$$

where $p$ is sub-gradient of $E$ at $u^{k}$. And $u, u^{k}$, and $u^{k+1}$ are the optimal $u, u$ in the $k$ th iteration, and $(k+1)$ th iteration respectively.

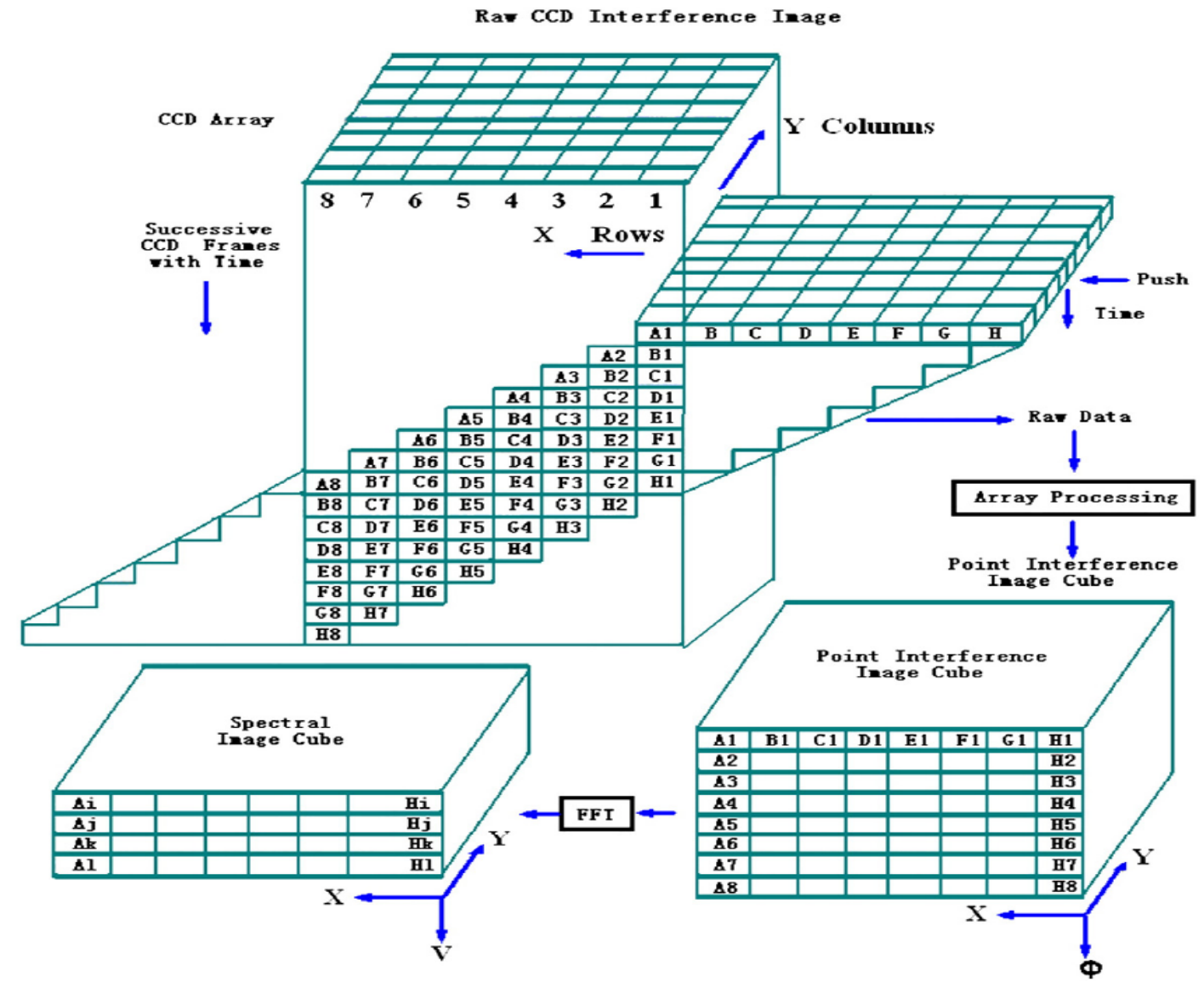

Fig. 3. Post-processing of LASIS 


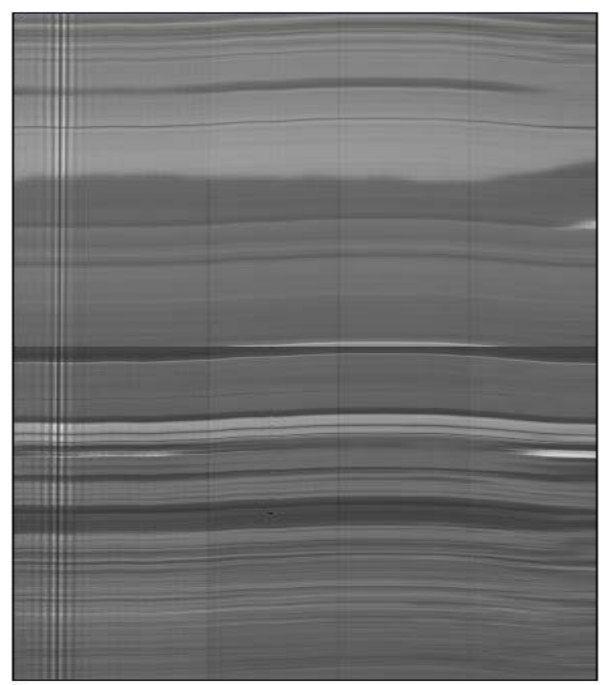

Fig. 4. One frame of LSMIS interference hyperspectral image

Two variables $d$ and $b$ (which can split the Bregman formulation and improve the efficiency of regularization by alternative minimization) are added into the split Bregman iteration, and alternative minimization of the two variables in formula (10) will be calculated to solve the problem in formula (7):

$$
\begin{aligned}
\left(u^{k+1}, d^{k+1}\right) & =\min _{u, d}|d|+E(u)+\frac{\lambda}{2}\left\|d-H(u)-b^{k}\right\|_{2}^{2} \\
b^{k+1} & =b^{k}+\left(H\left(u^{k+1}\right)-d^{k+1}\right)
\end{aligned}
$$

where $d$ and $b$ are two key variables in the split Bregman iteration, which can transform the original nonlinear problem into two linear problems of $d$ and $b$ respectively. The above transformation will improve the efficiency of original regularization of nonlinear problem. Basic steps of split Bregman iteration can be derived combining formula (9) with (10) and (11). The split Bregman iteration is widely used in the fields of image deblurring, basis pursuit, and image denoising [6-10]. The decomposition model for IHI data based on split Bregman iteration is first proposed in this paper, in order to get the optimal decomposed result with high efficiency.

\section{Image Decomposition Based on Split Bregman Iteration}

In this section, we will adopt split Bregman iteration to solve the problem of formula (6) and propose an efficient decomposition method for IHI data. As formula (6) has two 
regular terms, so four variables $d_{x}, d_{y}, b_{x}, b_{y}$, are introduced and formula (6) can be modified as the following formula (12) shows:

$$
\begin{aligned}
\left(X_{B}^{k+1}, d_{x}^{k+1}, d_{y}^{k+1}\right) & =\arg \min _{X_{B}, d_{x}, d_{y}} \frac{1}{2}\left\|X-X_{B}\right\|_{2}^{2}+\left|d_{x}\right|+\left|d_{y}\right| \\
+ & \frac{\lambda_{1}}{2}\left\|d_{x}-\nabla_{x} \otimes X_{B}-b_{x}^{k}\right\|_{2}^{2}+\frac{\lambda_{2}}{2}\left\|d_{y}-\nabla_{y} \otimes\left(X_{B}-X\right)-b_{y}^{k}\right\|_{2}^{2}
\end{aligned}
$$

Formula (12) will be solved through alternative minimization with respect to the three variables $X_{B}, d_{x}, d_{y}$, respectively. The exact steps are given as follows:

Step 1:

$$
X_{B}^{k+1}=\min _{X_{B}} \frac{1}{2}\left\|X-X_{B}^{k}\right\|_{2}^{2}+\frac{\lambda_{1}}{2}\left\|d_{x}^{k}-\nabla_{x} \otimes X_{B}^{k}-b_{x}^{k}\right\|_{2}^{2}+\frac{\lambda_{2}}{2}\left\|d_{y}^{k}-\nabla_{y} \otimes\left(X_{B}^{k}-X\right)-b_{y}^{k}\right\|_{2}^{2}
$$

Step 2:

$$
\begin{gathered}
d_{x}^{k+1}=\max \left(\left|\nabla_{x} \otimes X_{B}^{k+1}+b_{x}^{k}\right|-\frac{1}{\lambda_{1}}, 0\right) \cdot \frac{\nabla_{x} \otimes X_{B}^{k+1}+b_{x}^{k}}{\left|\nabla_{x} \otimes X_{B}^{k+1}+b_{x}^{k}\right|} \\
d_{y}^{k+1}=\max \left(\left|\nabla_{y} \otimes\left(X-X_{B}^{k+1}\right)+b_{y}^{k}\right|-\frac{1}{\lambda_{2}}, 0\right) \cdot \frac{\nabla_{y} \otimes\left(X-X_{B}^{k+1}\right)+b_{y}^{k}}{\left|\nabla_{y} \otimes\left(X-X_{B}^{k+1}\right)+b_{y}^{k}\right|} \\
b_{x}^{k+1}=b_{x}^{k}+\left(\nabla_{x} \otimes X_{B}^{k+1}-d_{x}^{k+1}\right) \\
b_{y}^{k+1}=b_{y}^{k}+\left(\nabla_{y} \otimes\left(X-X_{B}^{k+1}\right)-d_{y}^{k+1}\right)
\end{gathered}
$$

where $k$ is the iteration times of split Bregman, “*” in formula (14) and (15) refers to component-wise multiplication.

To get the optimal $X_{B}$ which will make the formula (13) has the minimization, the difference of formula (13) with respect to $X_{B}$ will be calculated and the following formula will be gotten:

$$
\begin{aligned}
& \left(1+\lambda_{1} \nabla_{x}^{T} \otimes \nabla_{x}+\lambda_{2} \nabla_{y}^{T} \otimes \nabla_{y}\right) \otimes X_{B} \\
= & X+\lambda_{1} \nabla_{x}^{T} \otimes\left(d_{x}-b_{x}\right)+\lambda_{2} \nabla_{y}^{T} \otimes\left(d_{y}+\nabla_{y} \otimes X-b_{y}\right)
\end{aligned}
$$

Let $T_{1}=X+\lambda_{1} \nabla_{x}^{T} \otimes\left(d_{x}-b_{x}\right)+\lambda_{2} \nabla_{y}^{T} \otimes\left(d_{y}+\nabla_{y} \otimes X-b_{y}\right)$,

$$
T_{2}=1+\lambda_{1} \nabla_{x}^{T} \otimes \nabla_{x}+\lambda_{2} \nabla_{y}^{T} \otimes \nabla_{y}
$$




$$
X_{B}=F^{-1}\left(F\left(T_{1}\right) . / F\left(T_{2}\right)\right)
$$

where $F$ and $F^{-1}$ are FFT (Fast Fourier Transformation) and inverse FFT respectively. ./ refers to component-wise division. Through calculating formula (19), we will get the optimal solution of formula (13) much more efficient.

The above operations in formulas (13) to (17) are only doing the Bregman iteration update for regularized terms. Considering the fidelity term $\frac{1}{2}\left\|X-X_{B}\right\|_{2}^{2}$ in formula (6), the original LSIMS IHI data $X$ will also need Bregman iteration update. Since the ideal background layer should not have too much difference with the original LSMIS image, so the update for fidelity term can be operated as follows:

$$
X^{k+1}=2 X_{B}^{k+1}-X^{k}
$$

Pseudo-code of proposed decomposition model is given as follows:

Input: LSMIS IHI image $X$, regularized weights $\lambda_{1}, \lambda_{2}$, two maximum iteration times of Bregman update (outer and inner) $N_{1}, N_{2}$, loop variable $k$

$k=0, \quad X_{B}^{k}=X$,

$d_{x}^{k}, d_{y}^{k}, b_{x}^{k}, b_{y}^{k}$ are the null matrices with the same size as $X$

for 1: $N_{1}$

for 1: $N_{2}$

$$
\begin{aligned}
& X_{B}^{k+1}=\min _{X_{B}} \frac{1}{2}\left\|X^{k}-X_{B}^{k}\right\|_{2}^{2}+\frac{\lambda_{1}}{2}\left\|d_{x}^{k}-\nabla_{x} \otimes X_{B}^{k}-b_{x}^{k}\right\|_{2}^{2}+\frac{\lambda_{2}}{2}\left\|d_{y}^{k}-\nabla_{y} \otimes\left(X_{B}^{k}-X^{k}\right)-b_{y}^{k}\right\|_{2}^{2} \\
& d_{x}^{k+1}=\max \left(\left|\nabla_{x} \otimes X_{B}^{k+1}+b_{x}^{k}\right|-\frac{1}{\lambda_{1}}, 0\right) \cdot \frac{\nabla_{x} \otimes X_{B}^{k+1}+b_{x}^{k}}{\left|\nabla_{x} \otimes X_{B}^{k+1}+b_{x}^{k}\right|} \\
& d_{y}^{k+1}=\max \left(\left|\nabla_{y} \otimes\left(X-X_{B}^{k+1}\right)+b_{y}^{k}\right|-\frac{1}{\lambda_{2}}, 0\right) \cdot * \frac{\nabla_{y} \otimes\left(X-X_{B}^{k+1}\right)+b_{y}^{k}}{\left|\nabla_{y} \otimes\left(X-X_{B}^{k+1}\right)+b_{y}^{k}\right|} \\
& b_{x}^{k+1}=b_{x}^{k}+\left(\nabla_{x} \otimes X_{B}^{k+1}-d_{x}^{k+1}\right) \\
& b_{y}^{k+1}=b_{y}^{k}+\left(\nabla_{y} \otimes\left(X-X_{B}^{k+1}\right)-d_{y}^{k+1}\right)
\end{aligned}
$$

end

$$
X^{k+1}=2 X_{B}^{k+1}-X^{k}
$$

$k=k+1$ 
end

Output: background layer $X_{B}$, interference stripe layer $X_{I}=X-X_{B}$

\section{Experiments and Analysis}

As Fig. 5 shows, 3 frames of LSMIS IHI data (12 bytes of size $256 \times 256$, the data came from Tianjin Key Laboratory of Optoelectronic Detection Technology and Systems, which are gotten after the post-processing [4] of the ground truth data LASIS IHI as Fig. 2-3 show) and one frame of LASIS IHI data (12 bytes of size $128 \times 128$ ) will be chosen for experimental comparison. The algorithms of IMCA and IMT will be adopted for comparison with the proposed method ( $\lambda_{1}, \lambda_{2}, N_{1}$ and $N_{2}$ are chosen to be 30, 500, 4 and 2 respectively). Compared results are shown as follows:

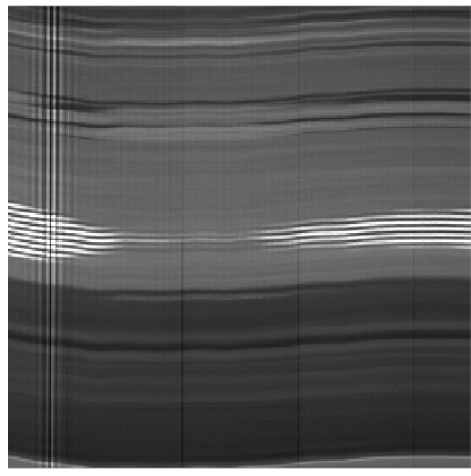

(a) LSMIS1

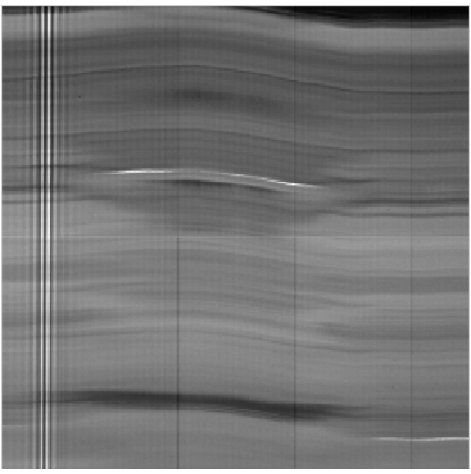

(c) LSMIS3

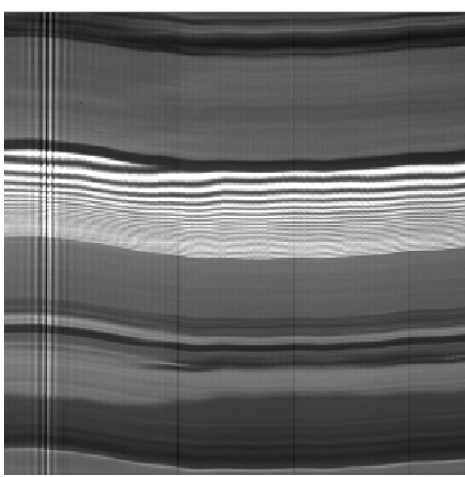

(b) LSMIS2

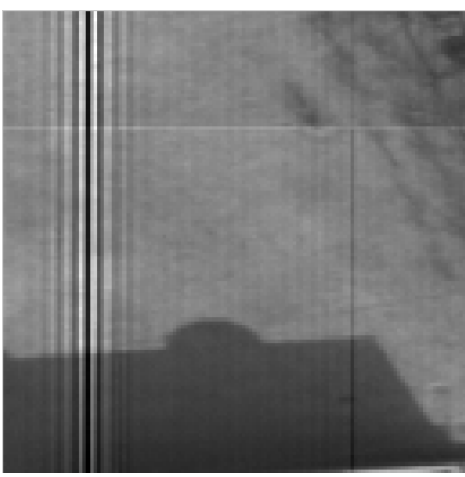

(d) LASIS

Fig. 5. Original LSMIS and LASIS IHI Image 


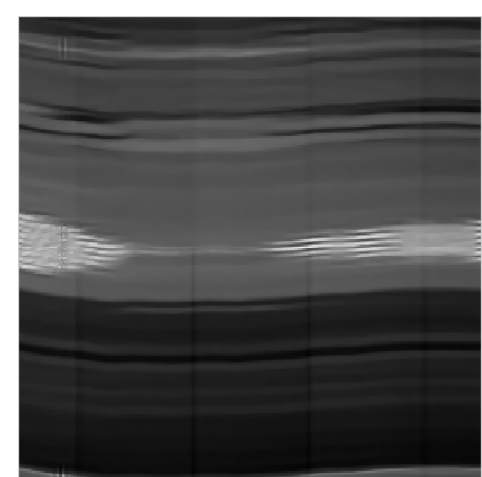

(a)

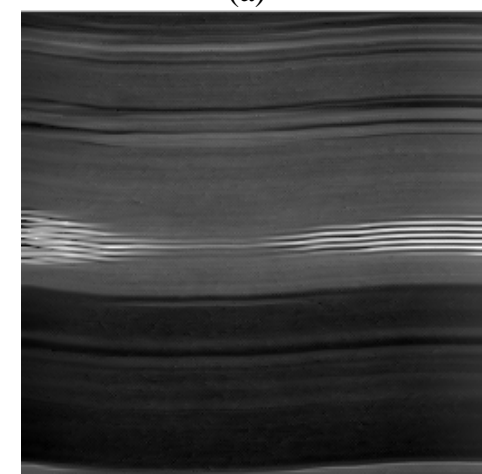

(c)

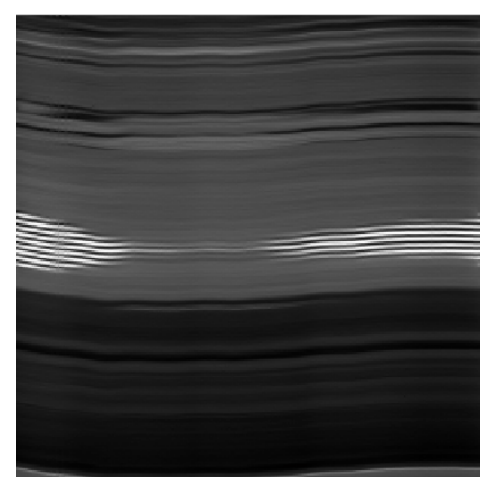

(e)

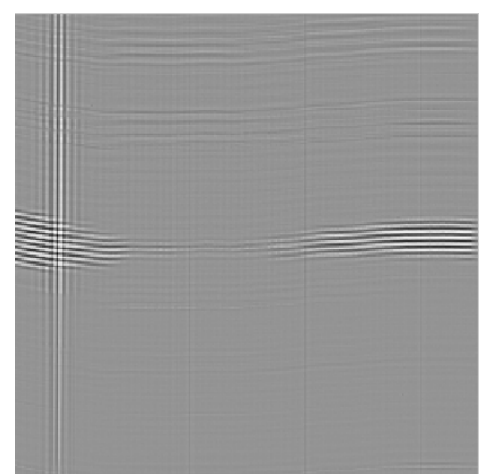

(b)

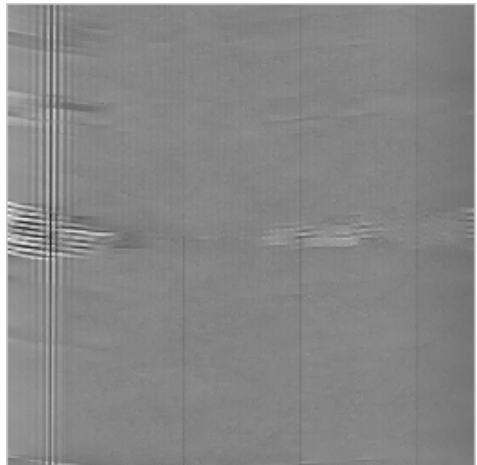

(d)

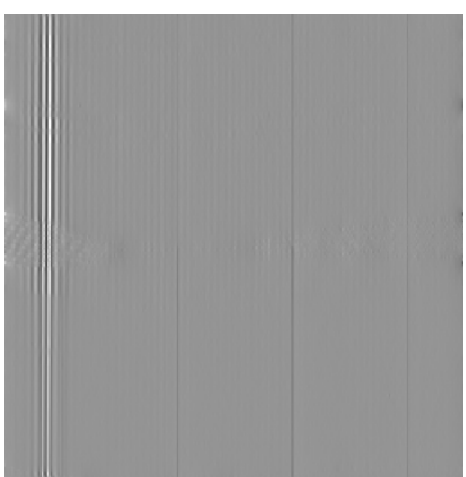

(f)

Fig. 6. Comparison Results of LSMIS1 Decomposition: (a) $X_{B}$ decomposed by IMCA (b) $X_{I}$ decomposed by IMCA(c) $X_{B}$ decomposed by IMT $<$ iteration $600>$ (d) $X_{I}$ decomposed by IMT $<$ iteration $600>$ (e) $X_{B}$ decomposed by proposed method (f) $X_{I}$ decomposed by proposed method 


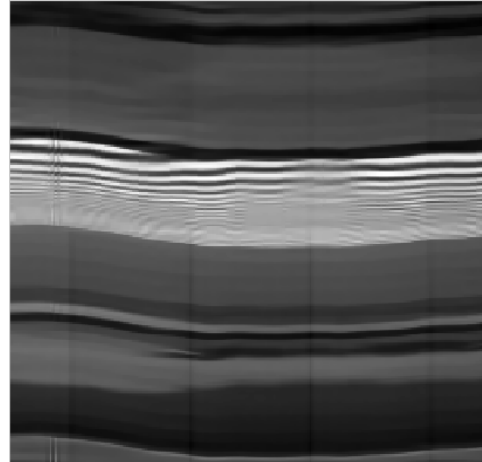

(a)

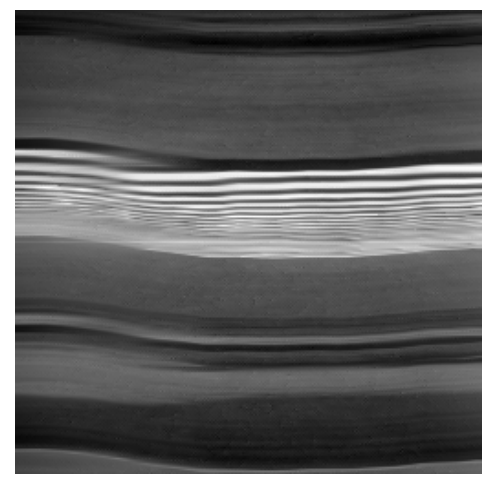

(c)
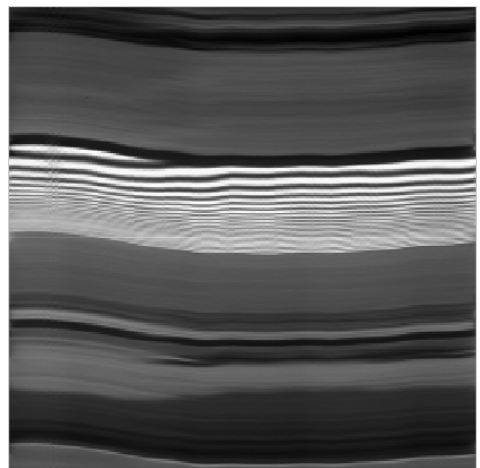

(e)

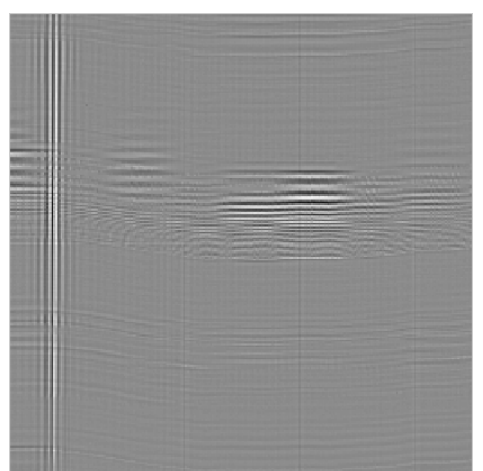

(b)

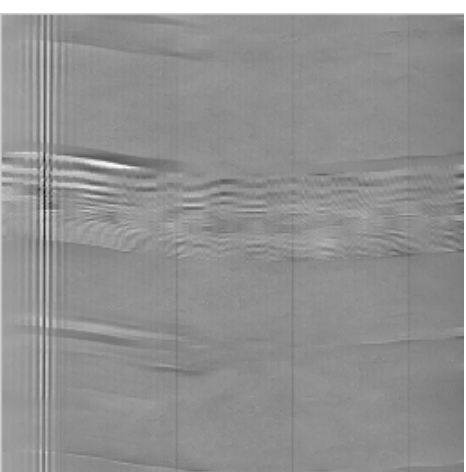

(d)

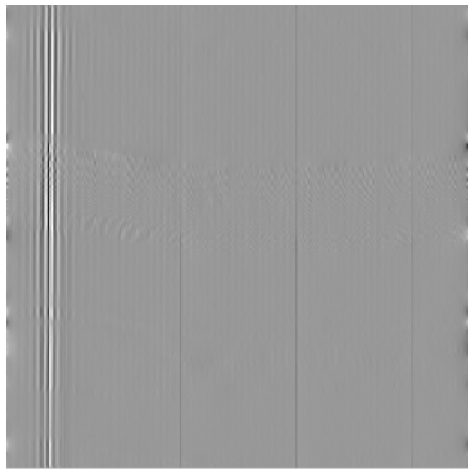

(f)

Fig. 7. Comparison Results of LSMIS2 Decomposition:

(a) $X_{B}$ decomposed by IMCA (b) $X_{I}$ decomposed by IMCA(c) $X_{B}$ decomposed by IMT<iteration $600>$ (d) $X_{I}$ decomposed by IMT<iteration $600>$ (e) $X_{B}$ decomposed by proposed method

(f) $X_{I}$ decomposed by proposed method 


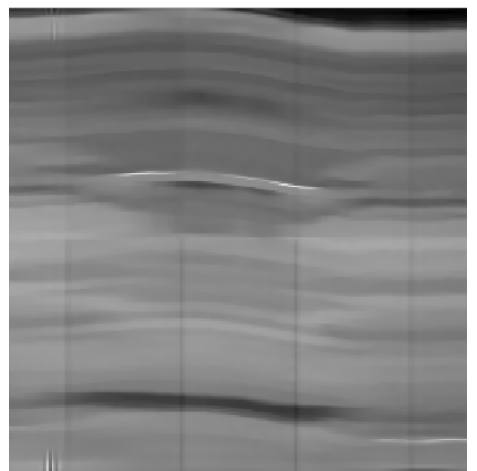

(a)

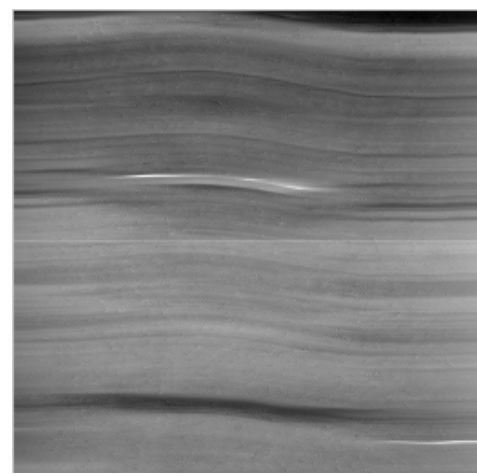

(c)

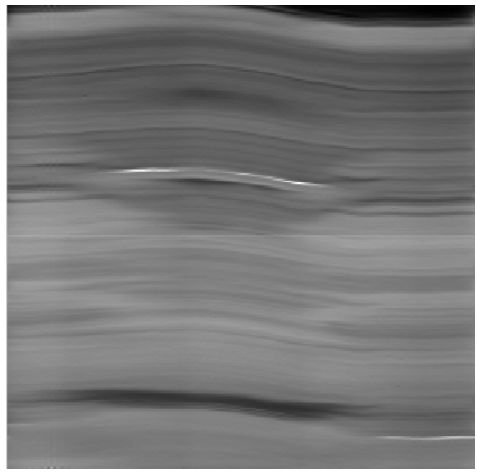

(e)

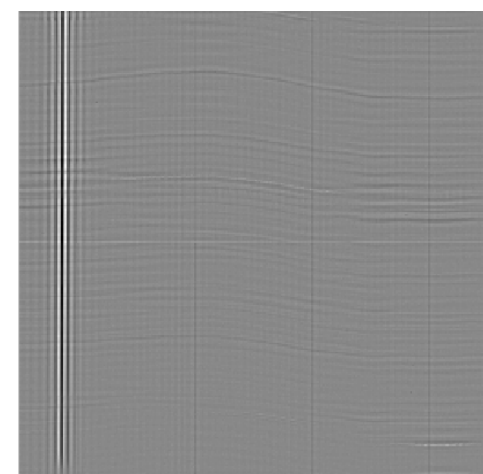

(b)

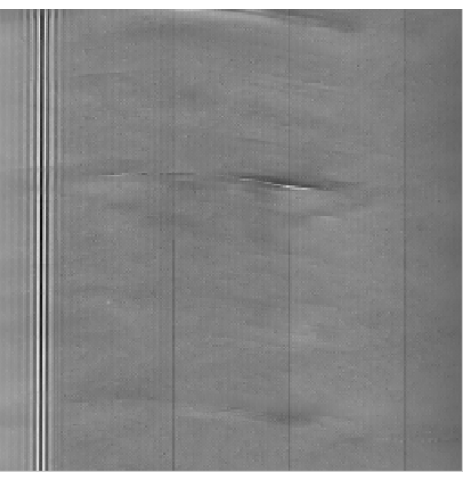

(d)

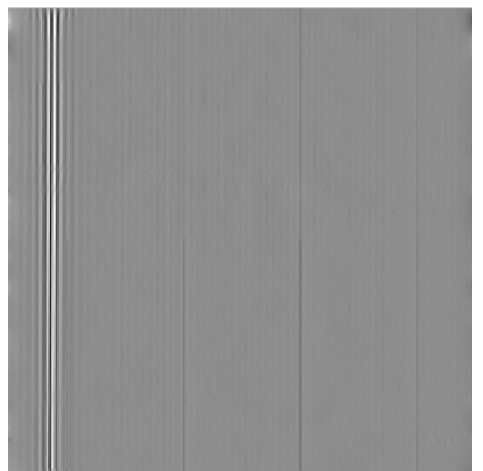

(f)

Fig. 8. Comparison Results of LSMIS3 Decomposition:

(a) $X_{B}$ decomposed by IMCA (b) $X_{I}$ decomposed by IMCA (c) $X_{B}$ decomposed by IMT $<$ iteration $600>$ (d) $X_{I}$ decomposed by IMT<iteration $600>$ (e) $X_{B}$ decomposed by proposed method

(f) $X_{I}$ decomposed by proposed method 


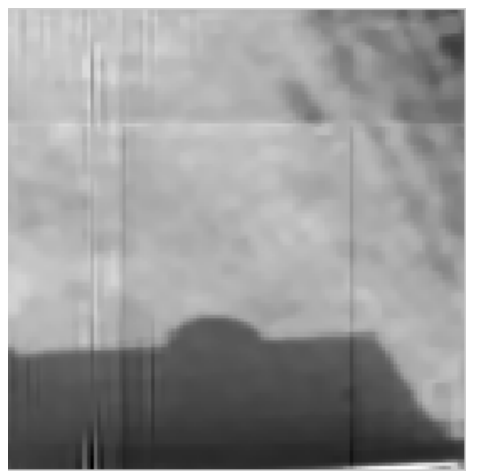

(a)

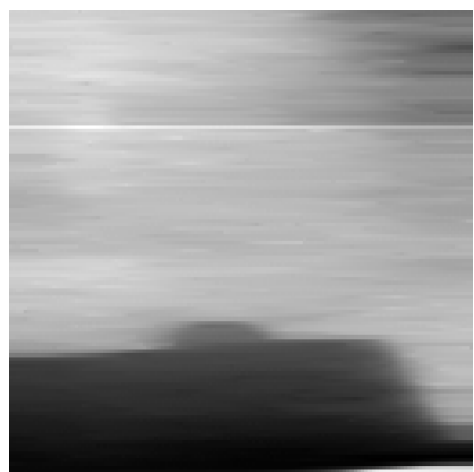

(c)

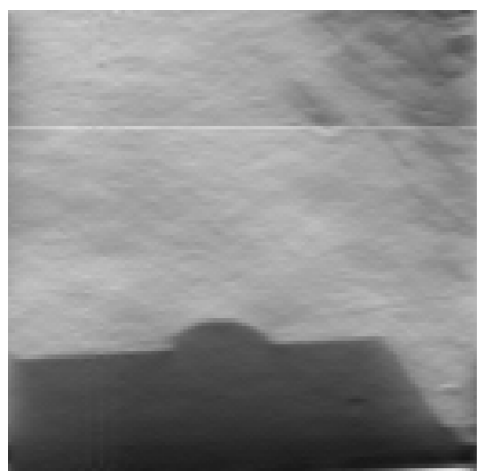

(e)

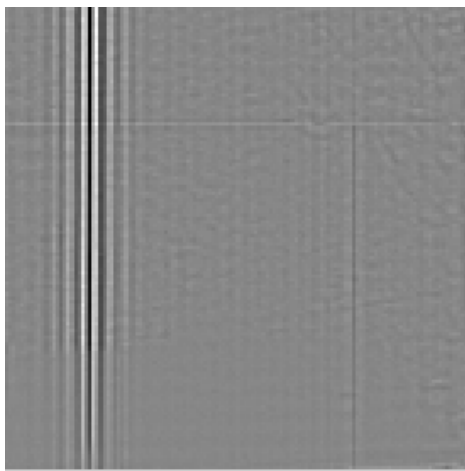

(b)

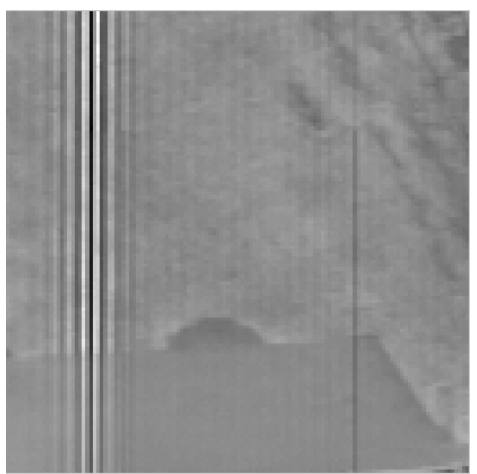

(d)

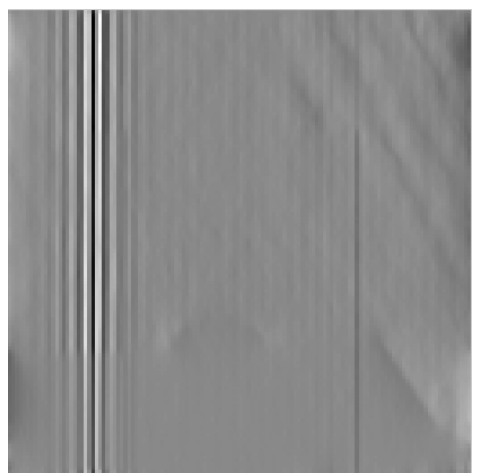

(f)

Fig. 9. Comparison Results of LASIS Decomposition:

(a) $X_{B}$ decomposed by IMCA (b) $X_{I}$ decomposed by IMCA(c) $X_{B}$ decomposed by IMT $<$ iteration $600>$

(d) $X_{I}$ decomposed by IMT<iteration $600>$ (e) $X_{B}$ decomposed by proposed method

(f) $X_{I}$ decomposed by proposed method 


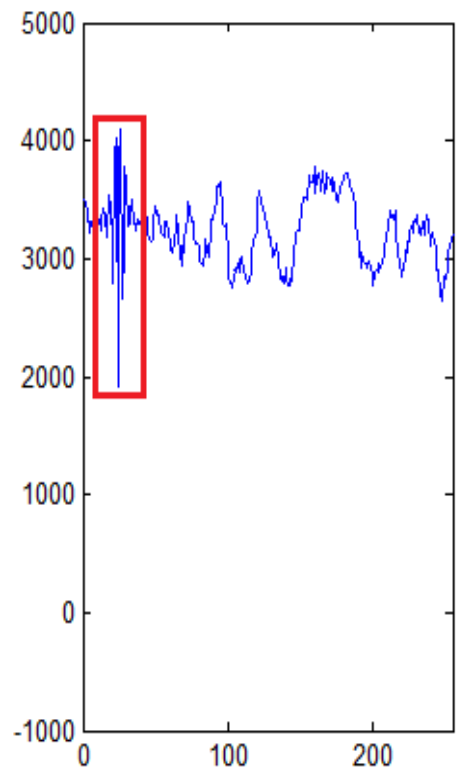

(a)

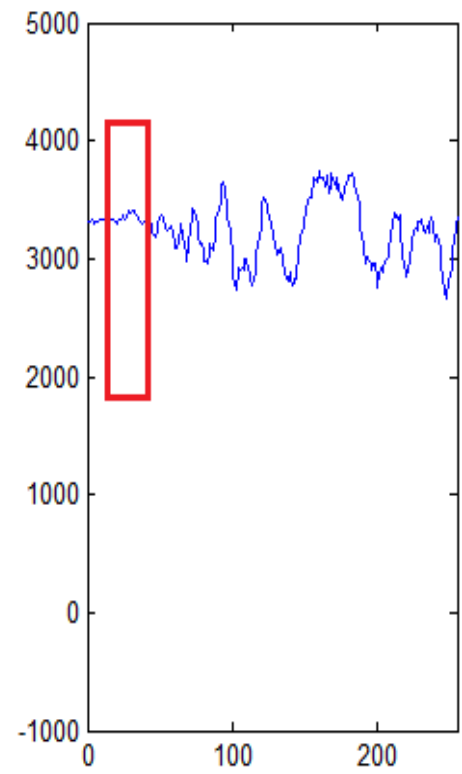

(b)

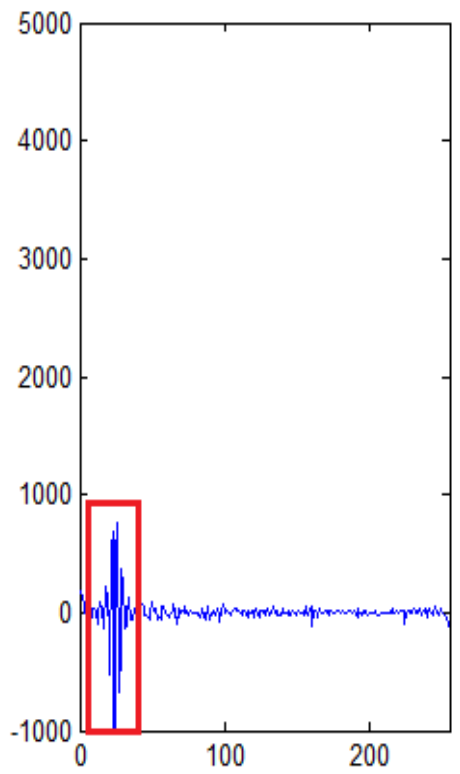

(c)

Fig. 10. Pixel values analysis after decomposition by proposed method: (a) Original LSMIS data (b) $X_{B}$ decomposed by proposed method (c) $X_{I}$ decomposed by proposed method

The quality of IHI image decomposition, the times of iteration, and the efficiency of the three methods are all calculated for comparison. Fig. 6-9 have shown the experimental results of LSMIS and LASIS IHI images decomposed by IMCA, IMT and the proposed method. From the experimental results, it is obviously seen that the background layers decomposed by IMCA still have residual interference stripes at the top and bottom, while the interference stripe layers decomposed by IMT are not clear enough. Compared with the above methods, background layer decomposed by the proposed method will be smoother, and the interference stripe layers decomposed by the proposed method will be cleaner enough with less background signals. Fig. 10 shows the decomposed results by means of the row-pixel values, and from the red frames (interference stripes part) we can obviously see the effect of the proposed method for LSMIS IHI decomposition, which can move the interference stripes from Fig. 10(a) to Fig. 10(c), and provide a clean background layer in Fig. 10(b).

Quantitative results of experimental comparison are shown in Table 1, to get the above visual effect, the iteration times needed for IMCA, IMT and proposed method are 15, 600 (Quantitative results of 400 iteration times for IMCA are also shown for comparison) and 8 respectively. The corresponding total variation of $X_{B}$ in horizontal direction and total variation of $X_{I}$ in horizontal direction are also shown. Quantitative results of Table 1 prove the proposed method not only can improve the quality of decomposed results, but only can reduce the iteration times significantly and make the decomposition much more efficient. 
Table 1. Quantitative Comparison of LSMIS IHI Decomposition

\begin{tabular}{|c|c|c|c|c|}
\hline & \multicolumn{4}{|c|}{ LSMIS1 } \\
\hline & IMCA & \multicolumn{2}{|c|}{ IMT } & Proposed \\
\hline Iteration times & 15 & 400 & 600 & 8 \\
\hline Runtime (s) & 13. 814790 & 9.768697 & 14.875874 & 0.436690 \\
\hline $\begin{array}{l}\text { Total variation of } X_{B} \text { in } \\
\text { horizontal direction }\end{array}$ & $7.3469 \mathrm{e}+06$ & $7.7183 e+06$ & $6.6424 \mathrm{e}+06$ & $9.3406 e+05$ \\
\hline $\begin{array}{l}\text { Total variation of } X_{I} \text { in } \\
\text { vertical direction }\end{array}$ & $3.1813 \mathrm{e}+07$ & $6.8651 \mathrm{e}+07$ & $6.6094 \mathrm{e}+07$ & $3.8866 \mathrm{e}+05$ \\
\hline \multirow[t]{3}{*}{$\begin{array}{c}\text { Value of the objective } \\
\text { function including the } \\
\text { fidelity term }\end{array}$} & $1.3069 \mathrm{e}+09$ & $1.7283 \mathrm{e}+10$ & $1.6627 \mathrm{e}+10$ & $1.1119 \mathrm{e}+08$ \\
\hline & \multicolumn{4}{|c|}{ LSMIS2 } \\
\hline & IMCA & \multicolumn{2}{|c|}{ IMT } & Proposed \\
\hline Iteration times & 15 & 400 & 600 & 8 \\
\hline Runtime (s) & 14. 127091 & 9.697143 & 15.425519 & 0.486476 \\
\hline $\begin{array}{l}\text { Total variation of } X_{B} \text { in } \\
\text { horizontal direction }\end{array}$ & $1.2998 \mathrm{e}+07$ & $1.0435 \mathrm{e}+07$ & $8.6132 \mathrm{e}+06$ & $1.3484 \mathrm{e}+06$ \\
\hline $\begin{array}{l}\text { Total variation of } X_{I} \text { in } \\
\text { vertical direction }\end{array}$ & $3.4853 \mathrm{e}+07$ & $1.0259 \mathrm{e}+08$ & $1.0003 e+08$ & $6.0367 \mathrm{e}+05$ \\
\hline \multirow[t]{3}{*}{$\begin{array}{l}\text { Value of the objective } \\
\text { function including the } \\
\text { fidelity term }\end{array}$} & $1.8741 \mathrm{e}+09$ & $2.5808 \mathrm{e}+10$ & $2.5141 \mathrm{e}+10$ & $1.7116 \mathrm{e}+08$ \\
\hline & \multicolumn{4}{|c|}{ LSMIS3 } \\
\hline & IMCA & \multicolumn{2}{|c|}{ IMT } & Proposed \\
\hline Iteration times & 15 & 400 & 600 & 8 \\
\hline Runtime (s) & 14.016324 & 9.823895 & 14.985148 & 0.430105 \\
\hline $\begin{array}{l}\text { Total variation of } X_{B} \text { in } \\
\text { horizontal direction }\end{array}$ & $5.1702 \mathrm{e}+06$ & $4.5709 \mathrm{e}+06$ & $3.1376 \mathrm{e}+06$ & $6.0186 \mathrm{e}+05$ \\
\hline $\begin{array}{l}\text { Total variation of } X_{I} \text { in } \\
\text { vertical direction }\end{array}$ & $3.2796 \mathrm{e}+07$ & $2.9492 \mathrm{e}+07$ & $2.8901 \mathrm{e}+07$ & $3.2986 \mathrm{e}+05$ \\
\hline \multirow[t]{3}{*}{$\begin{array}{l}\text { Value of the objective } \\
\text { function including the } \\
\text { fidelity term }\end{array}$} & $5.2830 \mathrm{e}+08$ & $7.4462 \mathrm{e}+09$ & $7.2769 \mathrm{e}+09$ & $9.1506 \mathrm{e}+07$ \\
\hline & \multicolumn{4}{|c|}{ LASIS } \\
\hline & IMCA & \multicolumn{2}{|c|}{ IMT } & Proposed \\
\hline Iteration times & 15 & 400 & 600 & 8 \\
\hline Runtime (s) & 3.905874 & 2.060725 & 3.117673 & 0.104174 \\
\hline $\begin{array}{c}\text { Total variation of } X_{B} \text { in } \\
\text { horizontal direction }\end{array}$ & $1.9134 \mathrm{e}+06$ & $1.0975 \mathrm{e}+06$ & $7.5844 \mathrm{e}+05$ & $1.9630 \mathrm{e}+05$ \\
\hline $\begin{array}{l}\text { Total variation of } X_{I} \text { in } \\
\text { vertical direction }\end{array}$ & $8.1270 \mathrm{e}+05$ & $3.8023 \mathrm{e}+06$ & $3.6252 \mathrm{e}+06$ & $1.0818 \mathrm{e}+05$ \\
\hline
\end{tabular}


Value of the objective function including the fidelity term

\section{Conclusions and Prospect}

Interference hyperspectral image data with high resolution, which can get the spectral and spatial information of the observed targets, has been widely used in many research fields. In this paper, a new method based on split Bregman iteration is proposed for decomposition of interference hyperspectral image data. The decomposition methods of IMCA and IMT are adopted for comparison with the proposed method. Experimental results have proved that the proposed method can reduce the iteration times which make the decomposition much more efficient than the existing decomposition methods.

\section{Acknowledgments References}

This work was supported by Tianjin Natural Science Foundation (No. 17JCQNJC01400), National Natural Science Foundation of China (Grant No. 61401439, 61601323) and Tianjin Science and Technology Major Projects and Engineering (17ZXSCSY00090)..

\section{References}

[1] Jia Wen, Caiwen Ma, Pengleng Shui, Yuli Xia, "Decomposition of Interference Hyperspectral Images Using Improved Morphological Component Analysis," Spectroscopy and Spectral Analysis, vol. 36, no. 1, pp. 254-258, July, 2015. Article (CrossRef Link)

[2] Jia Wen, Junsuo Zhao, Wang Cailing, "Improved MCA-TV algorithm for interference hyperspectral image decomposition," Optics and Lasers in Engineering, vol.75, pp. 81-87, 2015. Article (CrossRef Link)

[3] Jia Wen, Junsuo Zhao, Wang Cailing, "Improved morphological component analysis for interference hyperspectral image decomposition," Computers and Electrical Engineering, vol.46, pp 394-402, 2015. Article (CrossRef Link)

[4] Jia Wen, Caiwen Ma, Pengleng Shui, "An adaptive OPD and dislocation prediction used characteristic of interference pattern for interference hyperspectral image compression," Optics Communications, vol. 284, no. 20, pp. 4903-4909,2011. Article (CrossRef Link)

[5] Marouan Bouali, "Toward Optimal Destriping of MODIS Data Using a Unidirectional Variational Model," IEEE Transactions on Geoscience and Remote Sensing, vol. 49, no. 8, pp. 2924-2935, 2011. Article (CrossRef Link)

[6] Tom Goldstein, Stanley Osher, "The Split Bregman Method for L1-Regularized Problems," SIAM Journal on Imaging Sciences, vol. 2, no. 2, pp. 323-343, 2009. Article (CrossRef Link)

[7] JF Yang, Y Zhang, WT Yin. "An Efficient TV-L1 Algorithm for Deblurring Multichannel Images Corrupted by Impulsive Noise," SIAM Journal on Scientific Computing, vol. 31, no. 4, pp.2842-2865, 2009. Article (CrossRef Link)

[8] JF Yang, WT Yin, Y Zhang, Y Wang. "A Fast Algorithm for Edge-Preserving Variational Multichannel Image Restoration," Siam Journal on Imaging Sciences, vol. 2, no. 2, pp. 569-592, 2009. Article (CrossRef Link)

[9] Yonggui Zhu, Xiaoman Liu. "A fast method for L1-L2 modeling for MR image compressive sensing," Journal of Inverse and Ill-posed Problems, vol. 23, no. 3, pp.211-218, 2016. Article (CrossRef Link)

[10] JF Cai, Z Shen, "Linearized Bregman Iterations for Compressed Sensing," Mathematics of Computation, vol. 78, no. 267, pp.1515-1536, 2009. Article (CrossRef Link) 
[11] Yunyi Li, et.al., "SAITA: Sparse Adaptive-Iteratively-Weighted Thresholding Algorithm of Lp-Regularization using Multiple Sub-Dictionary Representation," Sensors, vol. 17, no. 12, pp. 1-17, 2017. Article (CrossRef Link)

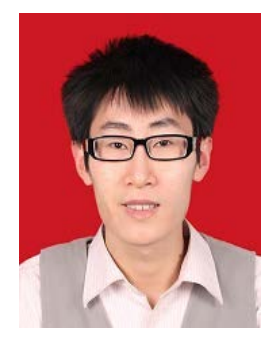

Jia Wen, Aug 1983, received the Bachelor and Master degree from Nanjing University, received the Ph.D. degree from Xi'an Jiaotong University. His research interests include sparse representation and compressive sensing, image and video processing.

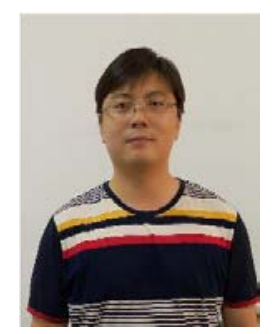

Lei Geng, received the Bachelor and Master degree from Shandong University, received the Ph.D. degree from Tianjin University. His research interests include image processing and pattern recognition.

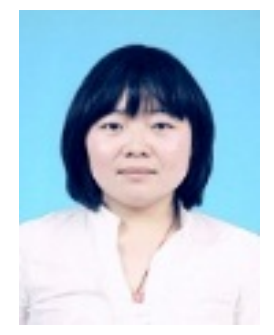

Cailing Wang(1984- ), female, lecturer, received the B.S. degree from Tianjin University in 2002, and the Ph.D. degree in Xi'an Institute of Optics and Precision Mechanics of CAS, Key lab of spectral imaging, Xi'an, China in 2011. From 2011, she is lecture in Xi'an Shiyou University. Her research interests in hyperspectral image classification and clustering. 